\title{
LES TREIZE POSTULATS DE L'INNOVATION PUBLIQUE : IDENTIFICATION ET DISCUSSION DANS LE CHAMP DE LA SANTÉ
}

\author{
Owen Boukamel et Yves Emery
}

De Boeck Supérieur | «Innovations »

2019/3 $\mathrm{N}^{\circ} 60$ | pages 15 à 41

ISSN $1267-4982$

ISBN 9782807392809

Article disponible en ligne à l'adresse :

https://www.cairn.inforevue-innovations-2019-3-page-15.htm

Distribution électronique Cairn.info pour De Boeck Supérieur.

(C) De Boeck Supérieur. Tous droits réservés pour tous pays.

La reproduction ou représentation de cet article, notamment par photocopie, n'est autorisée que dans les limites des conditions générales d'utilisation du site ou, le cas échéant, des conditions générales de la licence souscrite par votre établissement. Toute autre reproduction ou représentation, en tout ou partie, sous quelque forme et de quelque manière que ce soit, est interdite sauf accord préalable et écrit de l'éditeur, en dehors des cas prévus par la législation en vigueur en France. Il est précisé que son stockage dans une base de données est également interdit. 


\title{
Les treize postulats de l'innovation publique : identification et discussion dans le champ de la santé
}

\author{
Owen BOUKAMEL \\ Institut de hautes études en administration publique \\ Université de Lausanne (Suisse) \\ owen.boukamel@unil.ch \\ Yves EMERY \\ Institut de hautes études en administration publique \\ Université de Lausanne (Suisse) \\ yves.emery@unil.ch
}

\section{RÉSUMÉ}

Cet article vise à identifier et discuter les postulats véhiculés par la littérature sur l'innovation dans le secteur public. Il s'intéresse plus particulièrement aux organisations de santé. En se basant sur une revue de la littérature (98 études) sur l'innovation publique, treize postulats - affirmations sans preuve empirique - ont été identifiés. Ils portent sur la désirabilité de l'innovation et sur sa raison d'être (le pourquoi de l'innovation), sur sa nature (le quoi), sur son contexte (le où et le quand), et sur ses méthodes de développement (le comment et avec qui). Ces postulats se retrouvent dans la plupart des études analysées, que celles-ci soient consacrées au champ de la santé ou non. Or, le champ de la santé présente des spécificités qui mettent en doute la pertinence de ces postulats. C'est pourquoi ces derniers sont discutés à la lumière de ce champ.

MOTS-CLÉS : Innovation, Secteur public, Postulats, Santé, Critique

CODES JEL : H190

\section{ABSTRACT}

The Hidden Assumptions behind Public Sector Innovation: Implications for the Health Sector

This article aims to identify, and discuss the hidden assumptions of the literature on innovation of public organizations, with a special focus on studies 
about the health sector. Based on a systematic literature review (98 studies) we identified thirteen hidden assumptions on public sector innovation. These assumptions concern the desirability of public innovation and its legitimacy (the why of innovation), its nature (the what), its context (the where and when), and its methods of development (how and with whom). Our study shows that these assumptions are used in most studies, whether they are devoted to the health sector or not. However, the health sector has specificities that question the relevance of these assumptions. For this reason, the hidden assumptions are discussed in the light of this sector.

KEYWORDS: Innovation, Public Sector, Hidden Assumptions, Health, Critical Approach JEL CODES: H190

Cet article vise à identifier et discuter les postulats véhiculés par la littérature sur l'innovation des organisations publiques. Il s'intéresse plus particulièrement aux organisations actives dans le champ de la santé.

De nos jours, l'innovation revêt une connotation méliorative et il est courant de tenir un discours favorable à son développement, dans tout type d'organisation. Mais cela n'a pas toujours été le cas. Pendant des siècles, l'innovation était une menace pour la stabilité (Godin, 2015). Avec l'avènement de la révolution industrielle, puis l'émergence des théories schumpetériennes sur la destruction créatrice, l'innovation est devenue synonyme de modernité et de progrès. L'Etat n'a pas été épargné par cette tendance, à tout le moins depuis le début des années 2000. L'innovation est désormais une valeur constitutive de l'ethos public (Jorgensen, 2007).

Autour de cette question d'innovation publique, une littérature abondante s'est développée depuis une vingtaine d'années (de Vries, Bekkers, Tummers, 2016). Praticiens comme scientifiques affirment qu'aujourd'hui le secteur public n'a pas le choix : il doit innover. Toutefois, au motif de cet « impératif » d'innovation (Daglio, Gerson, Kitchen, 2015), cette littérature est souvent peu critique vis-à-vis de certains postulats fréquemment mobilisés. Ainsi, les motivations sous-jacentes à l'innovation, le bien-fondé et la valeur ajoutée des démarches soutenant l'innovation sont pris comme des acquis, des évidences qu'il n'est pas nécessaire de discuter.

Or tout questionnement scientifique devrait débattre de tels postulats le concept que nous allons retenir dans cette recherche - pour apprécier le développement de la connaissance dans un domaine précis, ici l'innovation publique. Ce constat s'opère tout spécialement pour le secteur de la santé, singulier à plusieurs égards : pluralisme des institutions, des métiers et des acteurs, complexité des technologies ou encore attentes fortes en termes de 
fiabilité et de continuité de service. Ces particularités font de la santé un champ complexe et spécifique du point de vue de l'innovation. Il est donc intéressant de discuter les postulats de l'innovation publique à la lumière des particularités de ce secteur édifiant pour notre analyse.

Le postulat est défini comme une "proposition que l'on demande d'admettre avant un raisonnement, que l'on ne peut démontrer et qui ne saurait être mise en doute ", ou encore comme un "principe de base, qui ne peut être mis en discussion $»^{1}$. Il ressort de ces définitions à la fois l'idée que le postulat devrait être pris comme une affirmation non discutable, au fondement de l'argumentation ou de la posture intellectuelle adoptée, et d'autre part que le postulat n'est pas (ou difficilement) démontrable. L'histoire de la connaissance et le développement des sciences fourmillent d'exemples qui illustrent le foisonnement de postulats, qui parfois s'érigent en paradigmes pris comme véridiques et définitifs (Kuhn, 1983). Or identifier et déconstruire ces paradigmes est à la base de la connaissance et du raisonnement scientifique, ce que Popper a défini comme la réfutabilité de la connaissance (Popper, 2005). Serionsnous entrés dans une période marquée par ce que nous pourrions appeler «le paradigme de l'innovation publique » (au sens de Kuhn (1983)), c'est-à-dire une période dans laquelle l'innovation publique est prise comme une évidence non démontrable et non questionnable, les seules énigmes à résoudre étant liées à la manière de susciter et développer l'innovation dans les organisations publiques?

Dans cette optique, les deux principales questions de la présente recherche sont les suivantes :

- Quels sont les postulats qui ressortent d'une part de l'examen de la littérature contemporaine sur l'innovation publique, et d'autre part de celle relevant spécifiquement du champ de la santé ?

- Comment ces postulats résonnent-ils dans le contexte de la santé : quels a priori et quelles conséquences engendrent-ils pour ce champ d'action publique?

Pour répondre à ces questions de recherche, cette étude ambitionne d'identifier et de décrire les principaux postulats présents dans la littérature sur l'innovation publique, en général et dans le champ de la santé plus spécifiquement. Puis dans un second temps, d'interroger et de déconstruire ces postulats en mobilisant plusieurs approches critiques issues de l'épistémologie, à la lumière des spécificités du champ de la santé.

1. Dictionnaire Larousse en ligne : https://www.larousse.fr/dictionnaires/francais/postulat/62959 (consulté le 16/04/2019). 
La première section revient plus en détail sur les éléments de cette problématique à travers un état de la recherche portant sur l'innovation publique en général, l'innovation dans le champ de la santé et sur le développement de la connaissance en lien avec la question des postulats. La deuxième section détaille la méthode d'identification des postulats, la troisième présente les résultats et la quatrième les discute dans le champ de la santé, en mobilisant des approches critiques complémentaires sur le développement de la connaissance.

\section{État de la recherche}

\section{L'émergence de l'innovation dans la littérature sur l’administration publique}

Depuis une vingtaine d'années, la littérature consacrée au phénomène d'innovation au sein du secteur public se développe à toute vitesse (de Vries et al., 2016 ; Taylor, 2018). Cette littérature doit son essor à l'idée selon laquelle l'administration ne devrait plus se contenter de soutenir l'innovation du secteur privé mais devrait innover par et pour elle-même. Les organisations publiques devraient développer une capacité d'innovation afin de répondre à une multitude de changements dans leur environnement, politiques, technologiques et sociaux, qui se manifestent également par les attentes nouvelles de leurs usagers, tout en devant faire face à des ressources (notamment budgétaires) durablement réduites (Borins, 2006 ; Boukamel, 2017 ; Osborne, Brown, 2011a).

Le développement de cette littérature est rapide comme le montre l'évolution du nombre de publications associées aux mots innovation public sector (Figure 1).

La littérature foisonne d'exemples d'organisations publiques innovantes (Townsend, 2013) et profitant des nombreux bienfaits (outputs) de l'innovation (de Vries et al., 2016).

\section{L'émergence d'une littérature sur l'innovation publique dans le champ de la santé}

La littérature sur l'innovation publique est plurielle. Elle s'intéresse à tous les domaines de politiques publiques (éducation, mobilité, citoyenneté, etc.), à tous les échelons (national, local, etc.), et à tous les types d'organisations (parapubliques, publiques, etc.). Au sein de ce grand ensemble, il est 
Figure 1 - Nombre de publications associées aux mots innovation public sector depuis 1999 (source : web of science, consulté le 5 septembre 2018)

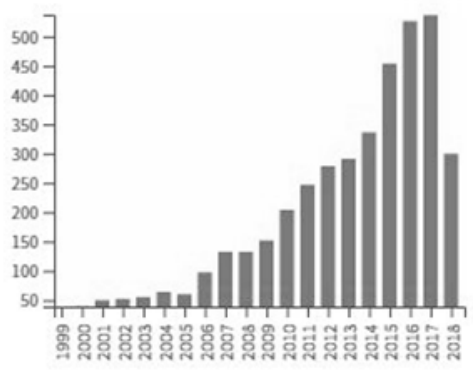

un corpus qui se penche plus spécifiquement sur l'innovation publique en prenant comme terrain de recherche le secteur de la santé ${ }^{2}$. À l'instar de la littérature générique, celle sur l'innovation dans le champ de la santé s'est développée rapidement. Sur les 636 publications se rapportant aux mots innovation health public sector publiés entre 2000 et 2018, environ la moitié (308) ont été publiées entre 2014 et $2018^{3}$. Cette littérature grandissante est très hétéroclite : elle va du management aux sciences du vivant en passant par les technologies médicales. Dans cette littérature, apparaissent des conceptions différentes de l'innovation, tantôt vue comme une opportunité d'adapter l'organisation ou le système en place, tantôt comme un moyen de faire mieux avec moins de ressources, tantôt comme le véhicule de réformes managériales. C'est notamment le cas pour les innovations de type processus managériaux qui sont souvent associées à la Nouvelle Gestion Publique (NGP) comme nous allons le voir (de Vries et al., 2016 ; Hansen, 2011).

\section{Les spécificités de l’innovation dans le contexte du champ de la santé}

La littérature sur l'innovation publique se développe donc, avec dans son sillage, celle sur l'innovation dans le champ de la santé. Néanmoins, ce champ présente de nombreuses particularités qui en font un espace de l'innovation singulier au sein des pays développés (OCDE, 2017).

Parmi les principales singularités, le champ de la santé est un espace marqué par un grand pluralisme des acteurs (administratifs, soignants, médicaux,

2. Parce qu'il est largement régulé par les politiques publiques, le champ de la santé est considéré comme appartenant au secteur public (au sens large), même si selon les pays la proportion d'acteurs public/privé peut varier sensiblement.

3. Source : Web of Science, consulté le 06.09.2018 
industriels et politiques), des institutions (hôpitaux, institutions de soins à domicile, etc.) mais également des objectifs (politique publique, expérience patient, contrôle des coûts, technicité, etc.) (Denis, Lamothe, Langley, 2001 ; Grenier, Bernardini-Perinciolo, 2015). Ce pluralisme complique les processus d'innovation, notamment parce qu'il complexifie la prise de décision (Friedland, Alford, 1991 ; Nobre, 2013).

Par ailleurs, le champ de la santé publique a été bouleversé par les principes et outils de la NGP et de manière générale, par les réformes relevant de la managérialisation (Rosenberg Hansen, Ferlie, 2016). En parallèle, les institutions de ce champ font les frais de pressions budgétaires, malgré l'ampleur des missions qui leur sont confiées (Cleeren, Lamey, Meyer, Ruyter, 2016). L'introduction de ces approches managériales et économiques suscite encore des tensions avec les logiques métier préexistantes, aux niveaux organisationnel et individuel (Grenier, Bernardini-Perinciolo, 2015).

Subséquemment à cette managérialisation et du fait de la cohabitation manifeste des acteurs publics et privés dans ce secteur, le champ de la santé est marqué par l'hybridité des organisations et des métiers (Gallouj, MerlinBrogniart, Moursli-Provost, 2015 ; Louise, Anneke, 2009) : les frontières entre les secteurs privé et public s'effacent progressivement (Christensen, Lægreid, 2011 ; Denis, Ferlie, Van Gestel, 2015 ; Emery, Giauque, 2014).

L'omniprésence des questions éthiques dans le champ de la santé s'ajoute à la complexité globale et à la singularité du champ vis-à-vis de l'innovation. Ces questions rendent, par exemple, la collecte de données, si utile à l'innovation, délicate (Vayena, Salathé, Madoff, Brownstein, 2015). Dans le même registre, la prise de risque, fréquemment associée à l'innovation (Boukamel, Emery, 2018 ; Osborne, Brown, 2011b ; Townsend, 2013) est épineuse dans les institutions de santé publique car nombre de leurs processus touchent la vie humaine.

Par ailleurs, l'ambidextrie contextuelle - qui consiste à impliquer tous les acteurs dans les activités d'exploitation mais également d'exploration ou d'innovation (Boukamel, Emery, 2017 ; O'Reilly, Tushman, 2013 ; Palm, Lilja, 2017) - semble difficile à développer dans les institutions de santé. Entre autres le manque de personnel, récurrent dans ce champ, pourrait contraindre les corps opérationnels à se consacrer exclusivement à l'exploitation et à délaisser l'innovation. Déconnectées de la conception, les personnes censées utiliser l'innovation ont parfois du mal à se l'approprier (Ologeanu-Taddei, Gauche, Morquin, Bourret, 2015).

Enfin, on peut mentionner la multiplicité des rythmes au sein de ce champ, entre le temps long de la recherche médicale et pharmaceutique et le temps court, voire urgent, des opérations quotidiennes. 
Toutes ces particularités font de la santé un champ complexe et particulièrement intéressant pour discuter des postulats identifiés dans la littérature sur l'innovation publique.

\section{Une littérature peu critique sur ses postulats}

\section{Le "comment " de l'innovation en dépit du "pourquoi "}

Le corpus de recherches théoriques et empiriques sur l'innovation des organisations publiques publié dans les revues internationales, est majoritairement constitué de contributions d'origines anglo-saxonne, scandinave ou néerlandaise. Ces recherches s'inscrivent avant tout dans des ontologies fonctionnalistes et positivistes. Le constat est le même pour la littérature sur l'innovation dans le champ de la santé publique, même si les contributions francophones (principalement françaises et québécoises) sont plus nombreuses. L'objectif fréquemment mentionné dans ces études est celui de comprendre comment l'innovation se développe dans les organisations publiques. Pour cette littérature, il paraît évident que l'innovation publique est à la fois nécessaire et positive. La forte présence de communications non académiques témoigne d'ailleurs de l'importance de cette problématique aux yeux des institutions et des consultants, tels que l'OCDE, ou les labs d'innovation publique (Daglio, Gerson, Kitchen, 2015 ; Mulgan, 2014 ; OECD, 2017). On y voit le résultat de l'association, implicite ou explicite, entre innovation et modernisation de l'Etat, couple indispensable pour relever les défis de la société contemporaine, comme en témoignent les paragraphes introductifs de très nombreux articles sur l'innovation publique (Damanpour, Schneider, 2009 ; de Vries et al., 2016). Sous l'impulsion des changements technologiques, la société se transforme et appelle en réponse apparemment inéluctable, un gouvernement digital (Digital era governement ou e-government (Dunleavy, Margetts, Bastow, Tinkler, 2005)) et une cyber-administration.

L'innovation semble donc non discutable et évidente et par essence neutre du point de vue axiologique, au sens de ne pas porter de valeurs spécifiques. La question de la finalité de l'innovation, le pourquoi innover dans l'administration publique, est souvent mise de côté. Or le développement de la connaissance devrait précisément éviter de prendre pour acquis des affirmations évidentes en apparence, mais plutôt les poser en hypothèses à démontrer (Popper, 2005). Le progrès de la connaissance est basé sur la validation ou l'invalidation d'hypothèses, qui sont au fondement de la démarche scientifique. Les impératifs de cette démarche apparaissaient déjà dans les travaux de Descartes, dont le premier principe de la Méthode est formulé ainsi : «ne recevoir jamais aucune chose pour vraie que je ne la connusse évidemment comme 
telle [...]» (1637). Dans cette optique, le postulat n'a pas de raison d'être, puisqu'une fois démontré par des études empiriques, il passerait à l'état de principe, de théorie ou de loi et ferait partie de la connaissance. Le caractère très fragile des postulats, mais également des principes et règles démontrés, apparait dans toute sa splendeur à travers l'histoire. Les sciences de l'épistémologie montrent bien comment les paradigmes dominants d'une époque imprègnent l'ensemble de la connaissance produite à ce moment et sont délaissés lorsqu'apparaît un nouveau paradigme.

La question du pourquoi de l'innovation dans l'administration publique est d'autant plus pertinente que l'innovation, la souplesse organisationnelle et l'adaptabilité prônées par cette littérature, sont des concepts qui peuvent entrer en dissonance avec les principes fondateurs de l'administration publique remontant aux travaux de Max Weber, parmi lesquels la contrôlabilité, la prédictibilité, et l'application stricte des règles qui régissent l'action publique, que seules les institutions démocratiques peuvent décider de modifier.

Aussi, dans cette recherche de la connaissance, nous souhaitons identifier et discuter les postulats, implicites ou explicites, qui apparaissent dans la littérature sur l'innovation publique.

\section{Une littérature construite en gap-spotting}

Il ressort que l'essentiel de la littérature sur l'innovation publique se construit de manière incrémentale, selon une logique de gap-spotting. Celle-ci consiste à identifier des manquements dans la connaissance et à les combler selon une stratégie de réflexion ad hoc. Une pratique qui n'interroge pas les postulats de base. Or, il est des auteurs qui montrent l'inanité du savoir scientifique lorsqu'il est érigé de cette manière, sans interroger les postulats fondateurs qui y sont mobilisés (Alvesson, Sandberg, 2011 ; Kuhn, 1983 ; Nizet, Pichault, 2015 ; Sandberg, Alvesson, 2011 ; Thietart, 2014). En lieu et place du gap-spotting, Alvesson, Sandberg (2011) proposent une construction de la connaissance découlant d'une problématisation, elle-même fondée sur l'identification et la discussion des postulats dominants d'un champ de connaissance.

\section{Un concept porteur de valeurs}

Quant à la neutralité du phénomène d'innovation dans les organisations publiques, nous l'avons dit, l'innovation est de nos jours clairement connotée positivement. Elle est porteuse, implicitement ou explicitement, d'amélioration (Godin, 2015). Cependant le progrès supposé de l'innovation doit être relativisé, car non seulement les résultats positifs escomptés ne sont pas toujours atteints, mais aussi parce que ces résultats dépendent bien souvent 
des acteurs qui peuvent se les approprier (Crozier, Friedberg, 1977), idée déjà présente dans la destruction créatrice de Schumpeter. Rouillard et Caron (2015) montrent de manière éloquente combien les innovations dans le secteur public peuvent, par exemple, aboutir à une détérioration des rapports et conditions de travail. D'autre part, l'innovation publique est fréquemment associée à la modernisation et au courant qui l'a portée ces vingt dernières années, à savoir la NGP (Pollitt, Bouckaert, 2011). Or, nombreux sont les auteurs qui soulignent que la modernisation liée à la NGP n'est pas neutre d'un point de vue idéologique (Bezes, 2008 ; Fortier, 2014 ; Hufty, 1998 ; Rouban, 2004). Dans la même veine, et même si les critiques sont moins nombreuses du fait de l'émergence récente de ce nouveau "paradigme ", la nouvelle gouvernance publique (Osborne, 2006) et le management par la valeur publique (Bryson, Crosby, Bloomberg, 2014) sont également sujets aux critiques (Chohan, Jacobs, 2017).

Au final et sans poursuivre ici une argumentation qui aurait pu être bien davantage étoffée, nous retenons à ce stade que l'innovation est manifestement porteuse de valeurs et que son caractère positif n'est pas toujours démontré par des recherches empiriques.

Ces réflexions critiques serviront à discuter les principaux postulats identifiés par la méthode suivie dans cette étude, qui est présentée dans le paragraphe suivant.

\section{Méthode}

Afin d'extraire les postulats dominants de la littérature sur l'innovation publique, il a fallu rassembler un corpus complet et pertinent d'articles scientifiques. Cette revue de littérature s'appuie sur la méthode de systematic review en quatre étapes, décrite par Grant, Booth (2009).

\section{Étape 1 : Exploration de la littérature}

Un large répertoire d'études sur l'innovation publique a tout d'abord été rassemblé. Pour ce faire, nous avons tout d'abord recherché des travaux sur les bases de données en ligne Web of Science (WOS), Science Direct (SD) et Google Scholar (GS), avec les mots-clés [innovat* public sector], puis [innovat* public services] car les deux dénominations peuvent se trouver dans la littérature anglophone ; ensuite en français [« innovation publique »] puis [« innovation services publics »] ; et enfin plus spécifiquement sur l'innovation dans le champ de la santé avec [public innovation health], l'idée étant d'identifier 
des postulats sur l'innovation publique du champ de la santé. Les résultats de cette première exploration de la littérature apparaissent dans le Tableau 1.

Tableau 1 - Recueil de références

\begin{tabular}{|l|l|l|l|l|l|l|}
\hline & Mots-clés & $\begin{array}{l}\text { Innovat* } \\
\text { public } \\
\text { sector }\end{array}$ & $\begin{array}{l}\text { Innovat* } \\
\text { public } \\
\text { services }\end{array}$ & $\begin{array}{l}\text { innovation } \\
\text { publique }\end{array}$ & $\begin{array}{l}\text { innovation } \\
\text { services } \\
\text { publics }\end{array}$ & $\begin{array}{l}\text { public } \\
\text { innovation } \\
\text { health }\end{array}$ \\
\hline \multirow{2}{*}{$\begin{array}{c}\text { Bases de } \\
\text { données en } \\
\text { ligne }\end{array}$} & $\begin{array}{l}\text { WOK } \\
\text { (recherche } \\
\text { par titre) }\end{array}$ & 277 & 247 & 1 & 0 & 131 \\
\cline { 2 - 7 } & $\begin{array}{l}\text { SD } \\
\text { (par mots- } \\
\text { clés }\end{array}$ & 92 & 133 & 0 & 0 & 0 \\
\cline { 2 - 7 } & $\begin{array}{l}\text { GS } \\
\text { (par titre) }\end{array}$ & 85 & 15 & 15 & 2 & 7 \\
\hline
\end{tabular}

Comme cette première recherche n'a engendré que peu de résultats sur l'innovation dans le champ de la santé et que ces résultats étaient principalement de type innovations médicales et pharmaceutiques, nous avons donc ciblé des revues d'Administration Publique. Nous avons sélectionné les cinq meilleures revues d'Administration Publique selon le Scimagojr $(H \text { index })^{4}$ : Administrative Science Quarterly, Journal of Public Administration Research and Theory, Public Administration Review, Public Administration et Journal of European Policy. Le mot-clef était [innovation healthcare]. Il est à noter que ce recueil complémentaire, se concentrant sur des revues anglophones, est susceptible d'engendrer une surreprésentation de références anglophones, ce qui constituerait une limite qu'il est d'ores et déjà utile de mentionner. Le résultat de cette démarche complémentaire apparaît dans le Tableau 2.

Tableau 2 - Recueil complémentaire de références

\begin{tabular}{|c|c|c|}
\hline \multirow{4}{*}{$\begin{array}{c}\text { Les cinq meilleures revues d'administration } \\
\text { publique }(\boldsymbol{H} \text { index })\end{array}$} & Mots-clés & $\begin{array}{c}\text { innovation } \\
\text { healthcare }\end{array}$ \\
\cline { 2 - 3 } \begin{tabular}{c} 
puld \\
\cline { 2 - 3 }
\end{tabular} & $\mathrm{ASQ}$ & 57 \\
\cline { 2 - 3 } & $\mathrm{JPART}$ & 24 \\
\cline { 2 - 3 } & $\mathrm{PAR}$ & 58 \\
\cline { 2 - 3 } & $\mathrm{PA}$ & 81 \\
\hline
\end{tabular}

À ce stade, nous avions ainsi rassemblé 1241 études.

4. https://www.scimagojr.com/journalrank.php?category=3321, recherche le 04.07.2018. Ces mêmes revues apparaissent en haut du classement pour les revues d'administration publique de la FNEGE : https:// fr.calameo.com/read/001930171df650a182b95. 


\section{Étape 2 : Critères d'inclusion}

Dans une deuxième phase, nous avons établi des critères formels destinés à sélectionner les études à inclure dans notre revue de littérature :

- Les études devaient être uniquement des articles de revues scientifiques pertinentes qui ont été révisés par les pairs.

- Ces articles devaient avoir été publiés entre 2000 et 2018, période reflétant le réel essor de l'innovation publique (de Vries et al., 2016).

- Ils devaient concerner uniquement l'innovation au sein du secteur public.

- Leur titre devait comporter le mot «innov*».

- Les articles devaient être rédigés en anglais ou en français.

\section{Étape 3 : Constitution du corpus d'études}

Nous avons tout d'abord exclu les doublons de la base de données. Ensuite, nous avons appliqué les critères d'inclusion sur la base du nom de l'article, de la date ou du statut de publication. De nombreux livres, thèses, communications de conférence, rapports ont été exclus. Ont été écartés par ailleurs, certains papiers traitant non pas de l'innovation publique mais de l'innovation privée soutenue par les politiques publiques. Enfin, les publications d'avant l'an 2000 ont été exclues. En troisième lieu, les critères d'inclusion ont été appliqués aux références restantes sur la base de la lecture des résumés et parfois des textes intégraux. Il est apparu notamment que certains articles étiquetés comme articles de revues scientifiques, s'avéraient être des opinions ou éditoriaux ou encore des recensions d'ouvrages.

Au final, sur les 1241 études rassemblées en premier lieu, nous en avons conservé 98 après avoir appliqué les critères d'inclusion. Ces études sont listées en Annexe 1.

Parmi ces 98 études, 21 se penchent théoriquement et/ou empiriquement plus spécifiquement sur l'innovation au sein du champ de la santé. Ce corpus dédié à la santé est majoritairement d'origine anglo-saxonne (les premiers auteurs de 9 sur les 21 études proviennent d'universités australiennes, américaines ou britanniques) ; mais aussi scandinaves (4 sur 21), allemandes ( 3 sur 21) ou encore francophones (2 sur 21). Notons que la prédominance des publications anglo-saxonnes reflète une partie de la réalité mais elle est aussi favorisée par notre recueil complémentaire de références, lequel se base sur les cinq revues les plus citées dans le champ, qui sont toutes anglo-saxonnes. Ces études sur la santé ont été publiées entre 2005 et 2018. Elles sont accolées d'une étoile (*) dans la liste des 98 références (Annexe 1). 


\section{Étape 4 : Identification des postulats}

Afin d'identifier les postulats, nous avons importé les 98 articles sélectionnés dans le logiciel Nvivo ${ }^{\circledR}$. Le codage s'est fait selon une stratégie inductive (Glaser, Strauss, 1967), à la suite d'un codage simultané de deux articles choisis aléatoirement dans le corpus et de leur mise en commun par les deux auteurs de la présente recherche. Cette stratégie de codage s'est établie en trois temps.

1. Tout d'abord nous sélectionnions les phrases qui allaient être codées. Pour ce faire nous recherchions le mot "innov* » dans les articles et gardions les cinq phrases qui avoisinaient le mot "innov* » au sein des articles, soit la phrase contenant le mot, les deux phrases avant et les deux phrases après. Cette technique nous a permis d'être plus efficaces pour cibler les postulats car nombreux étaient les extraits qui ne traitaient pas d'innovation.

2. Ensuite nous procédions à un codage primaire dans lequel nous codions en méta-nœud les concepts associés par les auteurs à l'innovation publique, sans considération sur le type de lien à ce stade (causalité, corrélation, etc.). Par exemple, les méta-nœuds issus de ce codage de premier ordre étaient : « technologie et innovation publique » ou « citoyens et innovation publique ».

3. Dans un troisième temps, nous appliquions un codage secondaire à l'intérieur de chacun de ces méta-nœuds afin de distinguer les postulats au sein d'une même thématique. Pour reprendre les exemples cités, les nœuds du méta-nœud « technologies et innovation publique » pouvaient être « la technologie entraine l'innovation publique » mais aussi « la technologie est un type d'innovation publique ».

Nous avons tout d'abord codé les articles non spécifiques au champ de la santé. Parmi ce corpus, 21 articles sélectionnés aléatoirement ont été codés avant d'arriver à saturation des arguments (Miles, Huberman, 1994). Ensuite, la totalité des 21 articles spécifiques à l'innovation dans le champ de la santé a été codée. Au total, 42 articles du recueil ont donc été traités. Les postulats identifiés dans ces deux corpus concentriques de littérature sont décrits dans la section suivante et discutés ensuite. Lors de l'identification des postulats, il est apparu que certains d'entre eux n'étaient pas partagés par tous les auteurs passés en revue. À l'inverse, certains postulats se retrouvaient partout. C'est pourquoi nous avons introduit dans notre analyse une appréciation qualitative de la fréquence à laquelle ces postulats se retrouvaient au sein de la littérature générique sur l'innovation publique, puis au sein de la littérature plus spécifique à la santé. 
Notons que cette méthode accepte une définition élargie du «postulat »: parfois des phrases identifiées comme des postulats se basent sur des références et ne sont donc pas, stricto sensu, des postulats. Pourtant, la présence d'une référence ne prouve pas formellement que l'affirmation avancée a été empiriquement vérifiée, certains travaux qui sont cités comme références n'apportent qu'un éclairage empirique partiel (ou alors il s'agit de contributions théoriques). Cette conception large du postulat peut être considérée comme une limite car elle engendre certainement des cas de faux-positifs (identifiés faussement comme des postulats), mais en contrepartie elle permet de ne rater aucun postulat (pas de faux-négatifs). Notons également qu'une analyse approfondie des postulats par sous-secteur du champ de la santé (public versus parapublic, ou hôpitaux versus autres secteurs) nous est impossible au regard du petit nombre d'articles qui composerait ces sous-secteurs. C'est pourquoi l'analyse porte sur les postulats du champ de la santé en général.

La littérature mobilisée pour la discussion critique de ces postulats emprunte ses idées aux approches critiques fondées sur les valeurs et les idéologies (Bezes, 2008 ; Fortier, 2010), celles d'obédience sociologique (Durand, Baret, Krohmer, 2018 ; Olivaux, 2016), et celles issues des Critical Management Studies (Willmott, 1992). Elles auront pour point commun une critique de la connaissance (Sandberg, Alvesson, 2011) dans le sillage de Kuhn (1983).

\section{Résultats}

Nous constatons tout d'abord que les postulats identifiés dans la littérature sur l'innovation dans le champ de la santé ne sont pas très différents des postulats issus de la littérature généraliste sur l'innovation publique. De plus, notre analyse montre que tous les articles du corpus partagent un postulat commun : l'innovation est synonyme de progrès. L'innovation publique serait substantiellement positive et donc désirable. Ce postulat est en quelque sorte un "postulat mère » car de celui-ci vont découler les treize autres postulats que nous avons identifiés.

Ces treize postulats ont été regroupés en quatre grandes «familles »:

1. les postulats portant sur la nature de l'innovation publique (le quoi),

2. ceux sur le contexte et l'histoire de l'innovation publique (les où et quand),

3. les postulats qui s'intéressent aux raisons d'être et à la légitimité de l'innovation publique (le pourquoi),

4. et enfin les postulats sur les méthodes et les acteurs du développement de l'innovation publique (les comment et grâce à qui). 
Tableau 3 - Synthèse des postulats identifiés dans la littérature sur l'innovation publique

\begin{tabular}{|c|c|c|c|c|}
\hline $\begin{array}{l}\text { Familles de } \\
\text { postulats }\end{array}$ & No. & Postulats & $\begin{array}{c}\text { Fréquence } \\
\text { dans la } \\
\text { littérature } \\
\text { générique } \\
\text { sur l'innovation } \\
\text { publique }\end{array}$ & $\begin{array}{c}\text { Fréquence } \\
\text { dans la } \\
\text { littérature sur } \\
\text { l'innovation } \\
\text { publique - } \\
\text { dans la santé }\end{array}$ \\
\hline $\begin{array}{l}\text { Le postulat mère: } \\
\text { L'innovation } \\
\text { est désirable }\end{array}$ & $\mathrm{O}$ & ...un progrès. & $* * *$ & $* * *$ \\
\hline \multirow{3}{*}{$\begin{array}{l}\text { Quoi ? } \\
\text { Les postulats } \\
\text { sur la nature } \\
\text { de l'innovation } \\
\text { publique }\end{array}$} & 1 & ...gérable. & $* *$ & $* * *$ \\
\hline & 2 & $\begin{array}{l}\text {...similaire à l'innovation } \\
\text { dans les services. }\end{array}$ & * & * \\
\hline & 3 & ...radicale. & * & Inexistant \\
\hline \multirow{3}{*}{$\begin{array}{l}\text { Où et Quand? } \\
\text { Les postulats } \\
\text { sur le contexte, } \\
\text { l'histoire et } \\
\text { la nature de } \\
\text { l'administration } \\
\text { publique } \\
\text { et de l'innovation }\end{array}$} & 4 & ...récente. & $* * *$ & ** \\
\hline & 5 & $\begin{array}{l}\text {...difficile dans } \\
\text { l'administration publique. }\end{array}$ & $* * *$ & $* * *$ \\
\hline & 6 & ...l'affaire du privé. & $* * *$ & $* * *$ \\
\hline \multirow{4}{*}{$\begin{array}{l}\text { Pourquoi ? } \\
\text { Les postulats } \\
\text { sur les raisons } \\
\text { d'être et la } \\
\text { légitimité de } \\
\text { l'innovation } \\
\text { publique }\end{array}$} & 7 & ...impérative. & *** & *** \\
\hline & 8 & $\begin{array}{l}\text {...capable de répondre } \\
\text { aux défis. }\end{array}$ & $* *$ & $* *$ \\
\hline & 9 & $\begin{array}{l}\text {...capable d'améliorer } \\
\text { l'efficience. }\end{array}$ & ** & $* *$ \\
\hline & 10 & $\begin{array}{l}. . . \text { le fruit d'une pression } \\
\text { externe essentiellement. }\end{array}$ & ** & $* *$ \\
\hline \multirow{3}{*}{$\begin{array}{l}\text { Comment et } \\
\text { grâce à qui ? } \\
\text { Les postulats } \\
\text { sur les méthodes } \\
\text { de développement } \\
\text { de l'innovation } \\
\text { et ses acteurs }\end{array}$} & 11 & $\begin{array}{l}\text {...à développer en s'inspirant } \\
\text { du secteur privé. }\end{array}$ & * & $*$ \\
\hline & 12 & $\begin{array}{l}\text {...portée par un groupe } \\
\text { d'acteurs. }\end{array}$ & ** & $* *$ \\
\hline & 13 & $\begin{array}{l}\text {...porté par une approche } \\
\text { collaborative. }\end{array}$ & ** & $* *$ \\
\hline
\end{tabular}

Ce découpage en familles structure cette section. Le Tableau 3 synthétise nos résultats en décrivant brièvement le postulat mère ainsi que les treize postulats de la littérature scientifique sur l'innovation publique. Pour chacun de ces postulats, nous précisons le degré de consensus qu'il présente dans la littérature. Certains des postulats sont présents dans la littérature mais rares $(*)$, d'autres sont plutôt fréquents $(* *)$ et enfin, certains sont très fréquents $(* * *)$.

\section{Quoi : les postulats sur la nature de l'innovation publique}

Les trois premiers postulats identifiés dans la littérature concernent la nature, la définition et l'essence de l'innovation publique. 


\section{$7^{\text {er }}$ postulat : l'innovation publique est gérable}

Le premier postulat consiste à considérer que l'innovation est mesurable et par conséquent gérable et maitrisable. Il s'oppose à l'idée que l'innovation serait spontanée et imprévisible. Ce postulat revient logiquement beaucoup dans les études qui analysent l'innovation en tant qu'extrant, mobilisant des intrants et produisant des effets tangibles. Il est fréquent dans la littérature sur l'innovation publique et très fréquent dans la littérature sur l'innovation dans le champ de la santé.

\section{$2^{e}$ postulat : I'innovation publique est simplement un type d'innovation de service}

Le deuxième postulat, moins consensuel que le premier, consiste à considérer que l'innovation publique n'est pas différente de l'innovation privée en cela qu'elle n'est simplement qu'une innovation de service. Cette posture tend à relativiser la frontière entre les secteurs public et privé et renforcer celle entre les innovations de service et les autres types d'innovation. Notons que ce postulat n'est pas partagé par toute la communauté scientifique et semble appartenir à une approche plus économiste de l'innovation.

\section{$3^{e}$ postulat : l'innovation publique est forcément radicale}

Le troisième postulat porte sur la radicalité de l'innovation publique. Selon ce postulat, l'innovation publique serait caractérisée par un changement radicalement nouveau, excluant de facto les évolutions incrémentales de la définition de l'innovation. Pour ces auteurs, si le changement incrémental était considéré comme une innovation, alors la frontière conceptuelle avec le concept de changement (ou d'amélioration) serait trop poreuse. Cela dit, à l'instar du précédent, ce postulat ne fait pas l'unanimité dans le champ de recherche, puisqu'il existe a contrario des études incluant bel et bien les innovations incrémentales dans le concept d'innovation. Il est même inexistant dans la littérature sur le champ de la santé.

\section{Où et quand : Les postulats sur le contexte, I'histoire et la nature de l'administration publique et de l'innovation}

Les trois postulats suivants ont trait au contexte dans lequel évolue l'idée d'innovation publique. 


\section{$4^{e}$ postulat : l'innovation publique est récente}

Selon ce postulat, le phénomène d'innovation au sein de l'administration publique (et non pas provenant de l'extérieur pour s'y implanter) serait récent. Plus précisément, il aurait véritablement commencé depuis le début des années 2000 environ. Les auteurs qui se basent sur ce postulat attribuent même ce phénomène à un mouvement global de renouvellement de l'administration publique et plus précisément à une période post-NGP, caractérisée par une distanciation avec les réformes managériales de l'administration qui ont marqué le dernier quart du $\mathrm{XX}^{\mathrm{e}}$ siècle. Alors que ce postulat est très fréquent dans la littérature générique, il l'est moins dans la littérature consacrée au champ de la santé.

\section{$5^{e}$ postulat : l'innovation publique est particulièrement difficile à implémenter dans l'administration publique}

Le cinquième postulat suppose que la « nature » de l'administration publique, par le biais de sa structure légale et bureaucratique, sa culture averse au risque, ses faibles incitations à innover et son ethos, serait intrinsèquement peu favorable à l'innovation. Pour cette raison l'innovation y serait particulièrement difficile à développer. Notons que ce postulat est particulièrement répandu dans la littérature.

\section{$6^{e}$ postulat : l'innovation est historiquement l'affaire du secteur privé}

Le sixième postulat affirme que l'innovation est historiquement un phénomène propre au secteur privé (cet argument entre en résonance avec l'argument précédent car le secteur privé aurait quant à lui, une nature favorable à l'innovation). Dans le secteur privé, l'innovation serait une question de survie dans un marché concurrentiel. C’est un postulat très fréquent.

\section{Pourquoi : Les postulats sur les raisons d'être et la légitimité de l’innovation publique}

Quatre postulats servent à légitimer l'existence d'innovation publique.

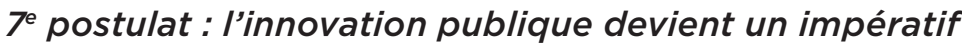

Pour de nombreux auteurs, l'administration serait confrontée à un ensemble de nouveaux défis sociodémographiques, économiques, écologiques et technologiques d'une ampleur inédite. Pour répondre à ces défis, l'innovation deviendrait un « impératif » pour les administrations publiques. Ce 
postulat de la légitimité de l'innovation est très répandu dans la littérature. Il apparaît dans la majorité des introductions d'articles.

\section{$8^{\mathrm{e}}$ postulat : I'innovation publique est capable de répondre aux défis posés à l'administration}

En lien avec le postulat précédent, affirmer que l'administration doit innover pour répondre à des défis nouveaux revient à également à faire le postulat que l'innovation en tant que telle est en mesure de répondre à ces défis, caractérisés par leur complexité croissante, voire pour certains auteurs par leur propension à être très difficiles à résoudre (Head, Alford, 2015).

\section{$9^{e}$ postulat : I'innovation publique est capable d'améliorer l'efficience de l'administration}

Parmi ces défis qui rendent « impérative » l'innovation publique, sont souvent mentionnés la pression budgétaire et les besoins d'efficience. Certaines études postulent que l'innovation serait capable d'améliorer l'efficience des organisations publiques.

\section{$10^{e}$ postulat : l'innovation publique est \\ essentiellement le fruit d'une pression externe}

Le dixième postulat identifié, en relation avec le «pourquoi de l'innovation ", laisse sous-entendre que l'innovation publique serait rendue impérative pour des raisons essentiellement exogènes, c'est-à-dire provenant de l'environnement des organisations publiques (les dits «impératifs »). Les innovations seraient bien moins le fruit d'impulsions provenant de l'intérieur.

\section{Comment et grâce à qui : Les postulats sur les méthodes de développement de l'innovation et ses acteurs.}

Enfin, trois postulats résident dans la manière avec laquelle les auteurs tentent de comprendre comment développer l'innovation publique et avec quels acteurs.

\section{$11^{e}$ postulat : l'innovation publique est à développer en s'inspirant du secteur privé}

Le onzième postulat identifié contient l'idée selon laquelle les organisations publiques devraient pour innover s'inspirer de pratiques émanant du secteur privé. Cette idée rejoint le sixième postulat qui attribue la paternité de l'innovation au secteur privé. Notons que ce postulat n'est que modérément 
répandu dans la littérature : il existe a contrario des auteurs qui revendiquent également une spécificité publique de la "gestion » de l'innovation (malgré tout, ces deux approches partagent le postulat premier : l'innovation publique est gérable).

\section{$12^{\mathrm{e}}$ postulat : l'innovation publique est portée par un groupe d'acteurs}

Le douzième postulat est fortement répandu et se base sur l'idée selon laquelle l'innovation publique serait portée par un groupe d'acteurs avant tout (tantôt les managers, les leaders, les employés publics ou encore des acteurs externes tels que des consultants, des think tanks, etc.). Gérer l'innovation consisterait entre autres à s'appuyer sur les capacités, compétences ou comportements de l'un de ces groupes d'acteurs.

\section{$13^{e}$ postulat : l'innovation publique est portée par une approche collaborative}

Enfin, le treizième postulat, issu d'une littérature plus récente, part du principe que l'innovation publique repose sur une approche ouverte de l'administration, qui mobilise notamment les collaborations entre acteurs et institutions. Les auteurs qui postulent cela ont tendance à inscrire également l'innovation publique dans une approche post-NGP et donc partagent le quatrième postulat. Notons que cette approche est particulièrement développée chez les auteurs d'Europe du Nord (notamment des Pays-Bas).

\section{Discussion}

Comme nous l'avons vu, nos résultats montrent que les postulats présents dans la littérature sur l'innovation dans la santé sont largement similaires à ceux véhiculés par la littérature générique. Cela malgré les spécificités du champ de la santé. Ce qui suggère que ces dernières sont peu prises en considération, ou qu'elles n'ont pas d'impact véritable sur le thème de l'innovation, ce qui serait pour le moins surprenant.

Dans les sous-sections suivantes, nous discutons donc les postulats identifiés dans la littérature à l'aulne des spécificités du champ de la santé. Au total, huit postulats sont discutés : certains d'entre eux car ils sont particulièrement peu pertinents dans l'étude de l'innovation au sein de la santé et donc que leur usage mériterait d'être questionné, d'autres, à l'inverse, sont discutés parce qu'ils pourraient être plus importants encore pour le champ de la santé qu'ils ne le sont ailleurs. 


\section{Le postulat mère $(0)$ et le contexte de la santé : l'innovation n'est pas toujours désirable}

Depuis les travaux de Schumpeter, l'innovation est vue comme un vecteur de progrès (Godin, 2015). Toutefois, si elle peut permettre des avancées, elle est rarement positive pour tous les acteurs concernés, surtout dans le domaine de la santé, sanctuarisé par les règles de l'éthique (Jordan, 2014). Trois exemples d'innovation nuancent le postulat de la désirabilité.

- La course aux équipements technologiques des organisations sanitaires peut parfois entraîner une tendance à surutiliser ces technologies afin de les rentabiliser.

- Les innovations qui concernent le management ou le financement du système sanitaire peuvent parfois nuire à la qualité des soins. C'est le cas, au moins en partie, des réformes qui ont introduit un financement par forfait fixé selon le diagnostic, aussi appelé tarification à l'activité ou Diagnosis-Related Group (DRG), et qui ont été présentées comme des innovations. Ces changements ont contribué à l'introduction de logiques de coûts et de compétitivité entre acteurs de la santé, ainsi qu'à une clientélisation du patient, créant in fine des tensions avec les logiques métiers de la chaîne de santé (Askfors, Fornstedt, 2018). De surcroît, cette recherche d'économie et d'efficience par pathologie traitée a pu contribuer à la réduction des durées de séjour hospitalier (Batifoulier, Castiel, Bréchat, 2017) et donc éventuellement à une baisse globale de la qualité des soins.

- Enfin, l'informatisation des pratiques médicales (comme par exemple la télémédecine, ou médecine à distance) peut concourir à la dépersonnalisation de la relation patient-soignant et donc à diminuer la qualité des soins du point de vue du patient (del Río Carral, Roux, Bruchez, Santiago-Delefosse, 2017).

Il apparaît ainsi que l'idée selon laquelle l'innovation est forcément désirable doit être nuancée, ad minima dans le champ de la santé, où les intérêts des acteurs en présence peuvent être très différents.

\section{Le postulat de la radicalité (3) de l'innovation difficile à concevoir dans le champ de la santé}

Dans le champ de la santé, les innovations radicales sont rares, notamment car les risques humains sont importants. L'innovation radicale y paraît délicate, à tout le moins lorsqu'en bout de processus se trouvent les patients. Par ailleurs, les activités médicales et de soins répondent la plupart du temps à des protocoles standardisés et une innovation radicale reviendrait à revoir 
ces protocoles sur toute la chaîne de soins et de formation des professionnels. À l'inverse, de nombreuses innovations dans le champ de la santé sont incrémentales, ad hoc, et décentralisées. C'est ce que suggèrent les auteurs du concept de « bricolage » dans les institutions de santé (Bloch, Bugge, 2013 ; Fuglsang, Sørensen, 2011). Ceci questionne les définitions de l'innovation qui se fondent sur la radicalité, car selon ces dernières, il n'y aurait pas ou prou d'innovation dans le champ de la santé. Il convient de s'interroger sur cette dissonance et, par ricochet, d'établir une définition de l'innovation incrémentale qui soit différente des concepts de changement ou d'amélioration. À cet égard, la définition de Rogers (2003) considérant innovant tout ce qui est perçu comme tel par les usagers (peu importe sa radicalité), semble être pertinente.

\section{Les postulats 4-5-11 à la lumière de l'éclatement des frontières entre les secteurs public et privé dans le champ de la santé}

Les quatrième (l'innovation est récente dans le secteur public), cinquième (l'innovation est plus difficile pour l'administration publique) et onzième (l'innovation publique doit être développer en s'inspirant du secteur privé) postulats partagent l'idée selon laquelle les secteurs public et privés sont substantiellement différents. Or, cette distinction est très peu pertinente dans le champ de la santé. En effet, ce dernier est marqué par l'hybridité des organisations et des métiers (Grenier, Bernardini-Perinciolo, 2015 ; Louise, Anneke, 2009). Cette hybridité a été introduite par les réformes de managérialisation; de manière naturelle par la cohabitation des organisations publiques et privées dans ce secteur ; par les logiques d'entreprise en vigueur (telle que la tarification à l'activité) ; ou encore par une certaine concurrence entre prestataires de soins. Par conséquent, cet effacement progressif des frontières entre les secteurs privé et public (Christensen, Lægreid, 2011 ; Denis et al., 2015 ; Emery, Giauque, 2014) questionne cette distinction de nature entre, d'un côté une innovation propre à l'administration publique et d'un autre, une innovation du secteur privé. Comme conséquence de cette inadéquation, il est probable que l'étude de l'innovation dans le champ sanitaire soit biaisée par la vision de l'espace trop vite assimilé à un espace spécifiquement public.

\section{Le postulat 4 à la lumière de la forte pénétration de la NGP dans le champ de la santé}

Le quatrième postulat, selon lequel l'innovation publique serait un phénomène récent lié au mouvement post-NGP, est présent dans le champ de la 
santé -même s'il l'est moins qu'ailleurs- et n'est pas tout à fait pertinent pour ce champ.

Il semblerait au contraire, que les innovations managériales et de processus dans le champ de la santé soient profondément marquées par la dynamique de NGP (Rosenberg Hansen, Ferlie, 2016) et par des coupes budgétaires les contraignant à une recherche continue de l'efficience (Cleeren et al., 2016). L'innovation serait un des vecteurs de cette dynamique de réformes initiée dans les années 1980 (Hansen, 2011) et particulièrement au RoyaumeUni ou des programmes nationaux ont encouragé les institutions à adopter des innovations managériales dans la lignée de la NGP (de Vries et al., 2016). L'innovation dans la santé paraît ainsi assez éloignée du mouvement récent de type post-NGP orienté vers la gouvernance, l'implication des stakeholders et la co-construction, même si elle peut naturellement s'en inspirer.

\section{Les postulats 12-13 à la lumière de la multitude d'acteurs qui compose le champ de la santé}

Il y a une autre catégorie de postulats qu'il est bon de discuter, non pas parce qu'ils ne correspondent pas à la réalité du champ de la santé, mais au contraire parce qu'ils s'y appliquent peut-être plus quailleurs, du fait de la multitude des acteurs présents. Il s'agit des douzième (l'innovation publique est portée par un groupe d'acteurs) et treizième (l'innovation est portée par les approches collaboratives) postulats. En effet, un grand pluralisme des acteurs (administratifs, soignants, médicaux, politiques, commerciaux) et des institutions (hôpitaux, institutions de soins à domicile, etc.) caractérise le champ de la santé (Denis et al., 2001). L'innovation publique dans ce champ semble donc être particulièrement tributaire de la collaboration et de l'implication large et efficace des acteurs, et ceci même si elle est portée par un groupe d'acteurs (douzième postulat), ce qui reste à prouver empiriquement.

\section{Conclusion}

Dans cette étude, nous sommes partis du constat selon lequel la littérature sur l'innovation publique serait en voie de devenir un colosse aux pieds d'argile. Alors qu'elle se développe à grande vitesse, ses fondations sont rarement questionnées, et donc fragilisées par toute une série de postulats. Dans le sillage de cette littérature générique, le corpus dédié à l'innovation dans le champ de la santé se développe également rapidement, et bizarrement, se fonde sur les mêmes postulats que la littérature sur l'innovation dans les autres secteurs. Dans cet article, nous avons cherché à montrer, à l'exemple 
du champ de la santé, que cette dynamique de construction de la recherche est risquée tant elle peut parfois s'éloigner de certaines réalités observables sur le terrain.

La première valeur ajoutée de cette étude se trouve dans l'identification des principaux postulats de la littérature sur l'innovation dans les organisations publiques génériques et dans celles de la santé. Ces postulats sont nombreux et attestent d'un certain nombre de croyances ou d'affirmations fortes, destinées à étayer le mouvement vers l'innovation, Mais il appartient à toute démarche scientifique de systématiquement questionner les postulats, explicites ou implicites, véhiculés par la littérature. L'estimation de la fréquence d'usage de chacun de ces postulats est aussi une valeur ajoutée de notre étude car elle montre que malgré la similitude entre les postulats des deux corpus, il existe des nuances dans la manière avec laquelle des postulats sont utilisés à l'intérieur d'un même champ d'étude. En quelque sorte, le corpus dédié à l'innovation publique dans le champ de la santé s'appuie sur les postulats de la littérature globale et les nuance légèrement. Par ailleurs, une valeur ajoutée théorique se trouve dans notre discussion des postulats identifiés. Celle-ci nous a amenés à nuancer la désirabilité de l'innovation : désirable peut-être, mais pour qui ? Et à quelles conditions ? Elle nous a aussi conduit à remettre sur le métier la définition de l'innovation qui, selon nos arguments, ne peut pas se fonder sur la simple radicalité. La définition de l'innovation devrait prendre en compte l'innovation incrémentale pour être complète. Un autre point soulevé dans cet article est celui de la pertinence de distinguer conceptuellement l'innovation publique et l'innovation privée. La porosité de certains domaines de l'administration avec les autres secteurs invite à repenser cette typologie. Enfin, notre article suggère que l'innovation, loin d'être un concept autonome, peut être le vecteur de valeurs déjà vieilles, à l'instar de celles véhiculées par la NGP.

Cette étude a aussi des limites. Parmi celles-ci, on peut citer le parti pris pour une définition élargie du "postulat ». En effet, de nombreuses phrases qui ont été codées au titre de postulat s'appuyaient sur des références scientifiques comme justification. Or, théoriquement un postulat n'en est pas un lorsqu'il dispose de justification empirique. Mais en pratique, une citation n'atteste pas toujours d'une preuve empirique : il peut s'agir d'une étude empirique ou d'un éclairage empirique que partiel. En fait, il est probable que chaque postulat se situe sur un continuum entre les deux extrêmes : aucune preuve empirique versus prouvé empiriquement. C'est pourquoi nous avons choisi de considérer ces affirmations référencées comme des postulats. Lavantage : nous évitons de passer à côté des postulats. L'inconvénient : nous nous exposons au risque d'avoir classé certaines affirmations empiriquement prouvées comme des 
postulats. En revanche nous n'avons jamais codé comme des postulats les résultats empiriques propres des articles analysés. Ce risque de faux-positif est donc modéré. Afin d'approfondir cet aspect, il aurait fallu remonter à la source de chacun des postulats identifiés, éventuellement à l'aide d'une carte de co-citations, en allant consulter les références mobilisées. Ce peut être l'objet d'une étude complémentaire.

La deuxième limite se trouve dans la sélection des articles qui composent la revue de littérature. Le choix des critères peut être discuté puisqu'il peut conduire à un biais de sélection. C'est notamment le cas des critères de langues : seules les études en anglais et dans une moindre mesure en français ont été intégrées. Or, il semble que plusieurs études dans d'autres langues soient intégrables à la vue du nombre de publications qu'elles représentent sur le thème de l'innovation publique (par exemple en néerlandais). Un autre critère discutable est le format et le statut des publications. En choisissant uniquement les articles revus par les pairs, il est possible que nous fussions passés à côté de postulats présents dans d'autres formats (comme par exemple les thèses ou les papiers de conférence). Le choix de la date de publication aussi (2000-2018) a peut-être engendré une surreprésentation des articles associant l'innovation et les mouvements de post-NGP massivement traités par la littérature dans cet intervalle et constituerait un biais. Subséquemment, afin d'approfondir l'analyse, il serait pertinent de mener des recherches qui élargissent les critères de sélection des articles (plus de langues, plus de statut de publications, plus d'années).

Enfin, une limite apparaît dans ce qui est entendu en tant qu'organisation de santé dans la littérature. En sélectionnant les articles traitant de la santé, indifféremment du secteur de la santé qu'ils concernaient (hôpitaux de dernier recours, cliniques, soins à domiciles, etc...), il est possible que notre analyse ait négligé une variabilité intra-sectorielle des postulats, liée à la diversité des contextes organisationnels que recouvre le champ de la santé. Saisir la variabilité intra-sectorielle dans notre analyse aurait nécessité un nombre d'études sur la santé (ici 21) nettement plus important. Lorsque la littérature sur le sujet sera plus développée, des études complémentaires pourraient ainsi s'atteler à partitionner ses sous-secteurs.

Au final, la présente contribution contribue modestement à porter un regard critique sur la littérature dédiée à l'innovation publique, et à rompre avec la spirale périlleuse de création de la connaissance sur des bases peu robustes. Si l'innovation paraît consubstantielle à la modernisation de la société et à celle, plus spécifique, de l'administration publique, également dans le domaine de la santé, il convient toutefois d'y porter un regard plus systématique, de l'ériger en problématisation plutôt qu'en gap-spotting (Sandberg, 
Alvesson, 2011), ceci afin d'éviter la tentation de la simplification, de la neutralité idéologique et de la pseudo-connaissance.

\section{RÉFÉRENCES}

ALVESSON, M., SANDBERG, J. (2011), Generating Research Questions through Problematization, Academy of Management Review, 36(2), 247-271.

ASKFORS, Y., FORNSTEDT, H. (2018), The Clash of Managerial and Professional Logics in Public Procurement: Implications for Innovation in the Health-Care Sector, Scandinavian Journal of Management, 34(1), 78-90.

BATIFOULIER, P., CASTIEL, D., BRÉCHAT, P.-H. (2017), La tarification à l'activité : une complexité contre-productive pour la santé des populations, Les Tribunes de la santé, 54(1), 61-78.

BEZES, P. (2008), Le tournant néomanagérial de l'administration française, in Borraz, O., Guiraudon, V. (dir.), Politiques publiques, Paris, Presses de Sciences Po, 215-254.

BLOCH, C., BUGGE, M. M. (2013), Public Sector Innovation: From Theory to Measurement, Structural Change and Economic Dynamics, 27, 133-145.

BORINS, S. (2006), The Challenge of Innovating in Government, 2nd edition, IBM center for the Business of Government, Washington, D.C.

BOUKAMEL, O. (2017), Le rôle des réseaux d'acteurs dans l'innovation publique complexe: le cas du vote électronique dans le canton de Genève, Politiques et Management Public, 34(3-4), 267-286.

BOUKAMEL, O., EMERY, Y. (2017), Evolution of Organizational Ambidexterity in the Public Sector and Current Challenges of Innovation Capabilities, The Innovation Journal: The Public Sector Innovation Journal, 22(2).

BOUKAMEL, O., EMERY, Y. (2018), Les freins culturels à l'innovation dans l'administration publique : Spécificités helvétiques, Gestion et Management Public, 6(4).

BRYSON, J. M., CROSBY, B. C., BLOOMBERG, L. (2014), Public Value Governance: Moving beyond Traditional Public Administration and the New Public Management, Public Administration Review, 74(4), 445-456.

CHOHAN, U. W., JACOBS, K. (2017), Public Value as Rhetoric: A Budgeting Approach, International Journal of Public Administration, 1-11.

CHRISTENSEN, T., LÆGREID, P. (2011), Complexity and Hybrid Public Administration, Theoretical and Empirical Challenges, Public Organization Review, 11(4), 407-423.

CLEEREN, K., LAMEY, L., MEYER, J. H., RUYTER, K. D. (2016), How Business Cycles Affect the Healthcare Sector: A Cross country Investigation, Health Economics, 25(7), 787-800.

CROZIER, M., FRIEDBERG, E. (1977), Lacteur et le système, Paris, Le Seuil.

DAGLIO, M., GERSON, D., KITCHEN, H. (2015), Building Organisational Capacity for Public Sector Innovation, OECD Conference. Innovating in the Public Sector : From Ideas to Impact, Paris, France. 
DAMANPOUR, F., SCHNEIDER, M. (2009), Characteristics of Innovation and Innovation Adoption in Public Organizations: Assessing the Role of Managers, Journal of Public Administration Research and Theory, 19(3), 495-522.

DE VRIES, H., BEKKERS, V., TUMMERS, L. (2016), Innovation in the Public Sector: A Systematic Review and Future Research Agenda, Public Administration, 94(1), 146-166.

DEL RÍO CARRAL, M., ROUX, P., BRUCHEZ, C., SANTIAGO-DELEFOSSE, M. (2017), Santé digitale : promesses, défis et craintes. Une revue de la littérature, Pratiques Psychologiques, 23(1), 61-77.

DENIS, J.-L., FERLIE, E., VAN GESTEL, N. (2015), Understanding Hybridity in Public Organizations, Public Administration, 93, 273-289.

DENIS, J.-L., LAMOTHE, L., LANGLEY, A. (2001), The Dynamics of Collective Leadership and Strategic Change in Pluralistic Organizations, The Academy of Management Journal, 44(4), 809-837.

DESCARTES, R. (1637), Discours de la méthode.

DUNLEAVY, P., MARGETTS, H., BASTOW, S., TINKLER, J. (2005), New Public Management is Dead: Long Life Digital-Era Governance, Journal of Public Administration Research and Theory, 16(3), 467-494.

DURAND, S., BARET, C., KROHMER, C. (2018), La sociologie de la traduction comme grille de recherche-intervention : le cas d'un projet de prévention des risques psychosociaux dans un hôpital public, RIMHE : Revue Interdisciplinaire Management, Homme E Entreprise, 30(1), 3-28.

EMERY, Y., GIAUQUE, D. (2014), The Hybrid Universe of Public Administration in the 21st Century, International Review of Administrative Sciences, 80(1), 23-32.

FORTIER, I. (2010), La réforme québécoise inspirée du managérialisme : description et enjeux, Revue française d'administration publique, 136, 803-820.

FORTIER, I. (2014), Ethos public et quête de sens dans cette ère de réforme : le NMP, ses critiques et les luttes pour la reconnaissance d'une spécificité du secteur public, Revue@ GRH, 9, 157-198.

FRIEDLAND, R., ALFORD, R. R. (1991), Bringing Society Back In: Symbols, Practices, and Institutional Contradictions, in Powell, W. W., DiMaggio, P. J. (eds), The New Institutionalism in Organizational Analysis, Chicago, USA, The University of Chicago Press, 232-263.

FUGLSANG, L., SØRENSEN, F. (2011), The Balance between Bricolage and Innovation: Management Dilemmas in Sustainable Public Innovation, The Service Industries Journal, 31(4), 581-595.

GALLOUJ, F., MERLIN-BROGNIART, C., MOURSLI-PROVOST, A.-C. (2015), Les partenariats public-privé et leur place dans l'innovation hospitalière : quels enseignements pour le management hospitalier ?, Innovations, 46(1), 161-195.

GLASER, B. G., STRAUSS, A. (1967), The Discovery of Grounded Theory: Strategies for Qualitative Research, Chicago, Aldine.

GODIN, B. (2015), Innovation: A Conceptual History of an Anonymous Concept, Working Paper, 2010-21 Project on the Intellectual History of Innovation. 
GRANT, M. J., BOOTH, A. (2009), A Typology of Reviews: An Analysis of 14 Review Types and Associated Methodologies, Health Information $\mathcal{E}$ Libraries Journal, 26(2), 91-108.

GRENIER, C., BERNARDINI-PERINCIOLO, J. (2015), Le manager hybride, acteur-passeur et acteur-clôture aux frontières institutionnelles. Analyse d'un médecin-chef de pôle hospitalier, Revue Française de gestion, 250(5), 125-138.

HANSEN, M. B. (2011), Antecedents of Organizational Innovation: The Diffusion of New Public Management into Danish Local Government, Public Administration, 89(2), 285-306.

HEAD, B. W., ALFORD, J. (2015), Wicked Problems Implications for Public Policy and Management, Administration $\mathcal{E}$ Society, 47(6), 711-739.

HUFTY, M. (1998), La pensée comptable. État, néolibéralisme, nouvelle gestion publique, Paris, PUF.

JORDAN, S. R. (2014), The Innovation Imperative: An Analysis of the Ethics of the Imperative to Innovate in Public Sector Service Delivery, Public Management Review, 16(1), 67-89.

JORGENSEN, T. B. (2007), Public Values, Their Nature, Stability and Change: The Case of Denmark, Public Administration Quarterly, 30(4), 365-398.

KUHN, T. S. (1983), La structure des révolutions scientifiques, Paris, Flammarion.

LOUISE, K., ANNEKE, F. (2009), Organisational Professional Conflict and Hybrid Clinician Managers: The Effects of Dual Roles in Australian Health Care Organisations, Journal of Health Organization and Management, 23(6), 642-655.

MILES, M. B., HUBERMAN, A. M. (1994), Qualitative Data Analyses: An Expanded Sourcebook, 2nd ed, CA, Sage.

MULGAN, G. (2014), Le design au service de l'innovation publique et sociale. Performance actuelle et améliorations possibles, traduit par La 27e Région.

NIZET, J., PICHAULT, F. (2015), Des positions épistémologiques aux positions institutionnelles, Les critiques de la gestion, Paris, La Découverte, 87-104.

NOBRE, T. (2013), L'innovation managériale à l'hôpital. Changer les principes du management pour que rien ne change ?, Revue Française de gestion, 235(6), 113-127.

O'REILLY, C. A., TUSHMAN, M. L. (2013), Organizational Ambidexterity: Past, Present, and Future, The Academy of Management Perspectives, 27(4), 324-338.

OCDE (2017), Panorama de la santé 2017, Paris, OECD Publishing.

OECD (2017), Fostering Innovation in the Public Sector, Paris, OECD Publishing.

OLIVAUX, M. (2016), Évolutions de l'hôpital public français : une approche interprétative par la culture organisationnelle. Le cas de deux centres hospitaliers universitaires, Thèse de doctorat, Université Aix-Marseille.

OlOGEANU-TADDEI, R., GAUCHE, K., MORQUIN, D., BOURRET, R. (2015), La capacité d'appropriation, une capacité organisationnelle immatérielle négligée dans l'adoption des systèmes d'information et de gestion, Innovations, 47(2), 79-100.

OSBORNE, S. P. (2006), The New Public Governance?, Public Management Review, 8(3), 377-387. 
OSBORNE, S. P., BROWN, L. (2011a), Handbook of Innovation in Public Services, Northampton MA, Edward Edgar Publishing.

OSBORNE, S. P., BROWN, L. (2011b), Innovation in Public Services: Engaging with Risk, Public Money $\mathcal{B}$ Management, 31(1), 4-6.

PALM, K., LILJA, J. (2017), Key Enabling Factors for Organizational Ambidexterity in the Public Sector, International Journal of Quality and Service Sciences, 9(1).

POlliTT, C., BOUCKAERT, G. (2011), Public Management Reform: A Comparative Analysis, Oxford, Oxford University Press.

POPPER, K. (2005), The Logic of Scientific Discovery, London, Taylor \& Francis.

ROGERS, E. M. (2003), Diffusion of Innovation, 5th Edition, New York Free Press.

ROSENBERG HANSEN, J., FERLIE, E. (2016), Applying Strategic Management Theories in Public Sector Organizations: Developing a Typology, Public Management Review, 18(1), 1-19.

ROUILLARD, C., CARON, I. (2015), Les nouvelles formes de domination dans la bureaucratie, in Emery, Y., Giauque, D. (dir.), L'acteur et la bureaucratie au XXIème siècle, Laval, PUL, 275-298.

SANDBERG, J., ALVESSON, M. (2011), Ways of Constructing Research Questions: GapSpotting or Problematization?, Organization, 18(1), 23-44.

TAYLOR, S. (2018), Innovation in the Public Sector: Dimensions, Processes, Barriers and Developing a Fostering Framework, International Journal of Research Science $\mathbb{E}$ Management, 5(1), 28-37.

THIETART, R. A. (2014), Méthodes de recherche en management, 4ème édition, Paris, Dunod.

TOWNSEND, W. (2013), Innovation and the Perception of Risk in the Public Sector, The International Journal of Organizational Innovation, 5(3), 21-34.

VAYENA, E., SALATHÉ, M., MADOFF, L. C., BROWNSTEIN, J. S. (2015), Ethical Challenges of Big Data in Public Health, PLoS computational biology, 11(2).

WILlMOTT, H. (1992), Critical Management Studies, Sage Publications (CA). 